\title{
Measuring plant disease severity in R: introducing and evaluating the pliman package
}

\author{
Tiago Olivoto ${ }^{1 *}$, Sheila M. P. Andrade ${ }^{2}$, Emerson M. Del Ponte ${ }^{2}$ \\ ${ }^{1}$ Departamento de Ciências Agronômicas e Ambientais, Universidade Federal de Santa Maria, \\ 99840-000 Frederico Westphalen, RS, Brazil \\ ${ }^{2}$ Departamento de Fitopatologia, Universidade Federal de Viçosa, 36570-900 Viçosa, MG, Brazil \\ *Correspondence: Tiago Olivoto <tiagoolivoto@gmail.com>
}

\section{Abstract}

Image analysis based on color thresholding is the reference method for measuring severity as percent area affected. It is deemed to produce accurate results, usually considered the "true" severity value. More than a dozen applications have been used for the task in phytopathometry studies, but none was coded in R language. Here we introduced and evaluated pliman, a suite for the analysis of plant images. In particular, we show functions for computing percent severity based on RGB information contained in image palettes prepared by the user. Six image collections, totaling 249 images, from different diseases (wheat tan spot, soybean rust, olive leaf spot, rice brown spot, bean angular spot, and Xyllela fastidiosa on tobacco) exhibiting a range of symptomatic patterns and severity were used to evaluate the agreement of pliman predictions with APS Assess, LeafDoctor and ImageJ. Three users independently prepared three image palettes (each representing leaf background, symptomatic or healthy leaf tissue) by manually inspecting and subsetting these target areas in the images. Pliman predictions by a joint palette (by joining images by the three users into one) were highly concordant $\left(\rho_{c}>0.98\right)$ with measures by the other software for all but Xylella fastidiosa on Tobacco $\left(\rho_{c}=0.49\right)$. The error for the latter may be due to the low contrast between symptomatic and healthy tobacco tissues. Users showed to be a source of variation in the overall concordance depending on the disease. Reduction in the image resolution $(<1$ megapixel) did not impact the results. Combined with parallel processing, the use of low image resolution sped up the processing time resulting in pliman being 170 to 430 times faster than existing tools for disease quantification. Pliman showed great potential to produce accurate measures and accelerate studies involving plant disease severity measurements, especially for the batch processing of large sets of image collections.

Keywords: Phytopathomery; Plant disease assessment; Image processing 


\section{$\because$ OSFPREPRINTS \\ https://doi.org/10.31219/osf.io/jfhm7}

Introduction

In phytopathometry, the term severity is commonly referred to as the relative area of a sampling unit (plant surface) affected by disease symptoms or signs (Nutter et al. 1991). It is a quantitative variable and one considered essential for many purposes including monitoring disease epidemics, predicting yield loss, comparing plant phenotypes, and evaluating effects of treatments on disease (Nutter and Gaunt 1996; Madden et al. 2007; Bock et al. 2010). Historically, and still very commonly, especially in field operations, percent severity is obtained via visual assessment, with or without aids such as standard area diagrams (SADs) (Bock et al. 2021).

As an alternative to visual assessments, image analysis has been used for several decades for measuring severity (Lindow and Webb 1983). However, it was only during the mid-2000s that the first plant disease-specific image analysis programs were made available (Lamari 2002; Vale et al. 2003). The color thresholding method for manually annotating plant disease severity is considered the best approximation to obtaining the "true" value of severity (Bock et al. 2020). Currently, more than a dozen software, most of them of commercial license, and based on color-thresholding, have been used for manually segmenting areas of plant disease images that are determined as background, symptomatic, and nonsymptomatic (Del Ponte et al. 2017). Because the manual annotation for large numbers of images may be laborious and time-consuming, macros have been made available in some software including Assess (Lamari 2008), ImageJ (Schneider et al. 2012), and Quant (Vale et al. 2003), or automatic methods have been developed for the purpose (Barbedo 2016a). Besides their use in research conducted at more controlled conditions, usually at a smaller scale, these softwares have been especially useful for the development and validation of standard area diagrams (SADs) for which the actual value is needed for the diagrams and the full set of images used during SADs validation studies (Del Ponte et al. 2017).

The objective of this article is to introduce and evaluate the accuracy of severity determined using custom functions of the R package pliman (short for plant image analysis). The functions were built specifically for modeling and predicting disease severity on a target image based on RGB information contained in image palettes (subsections of the target 


\section{$\because$ OSFPREPRINTS \\ https://doi.org/10.31219/osf.io/jhm7}

image) prepared by the user. Additionally, we aimed at evaluating whether the reduction in image resolution affected the results as well as the computation time.

\section{Material and Methods}

\section{The pliman $\mathbf{R}$ package}

The pliman package is a suite for performing several analyzes in images of plants, especially related to foliar analysis, including: measuring leaf area, counting objects in an image, computing disease-symptomatic area, extracting the RGB values for each object in an image, computing object measures. The package is built on the existing image analysis package EBImage (Pau et al. 2010). The current stable version of the package (0.3.0) requires $R$ 4.1.0 and can be installed from CRAN directly via the R console using:

install.packages ("pliman")

The development version of the package is available on Github and can be installed (devtools package should be previously installed) using:

devtools: :install_github ("Tiagoolivoto/pliman")

\section{Computing disease severity with pliman}

One of the functions in pliman for computing percent disease severity is the symptomatic_area(), which processes a single or multiple images and returns a data.frame object with at least two values: percent of healthy and percent of symptomatic area in the processed image. A simplified workflow from image import, processing, and severity prediction is presented in Figure 1.

In brief, the computation is based on color information extracted from each of three palettes representing different subareas of the images, or classes: 1 ) leaf background (B), and the 2 ) symptomatic $(S)$, and 3 ) healthy $(H)$ areas of the leaf. The image should have a solid background color (e.g. blue color) that contrasts with the leaf and disease symptom colors. 
https://doi.org/10.31219/osf.io/jfhm7

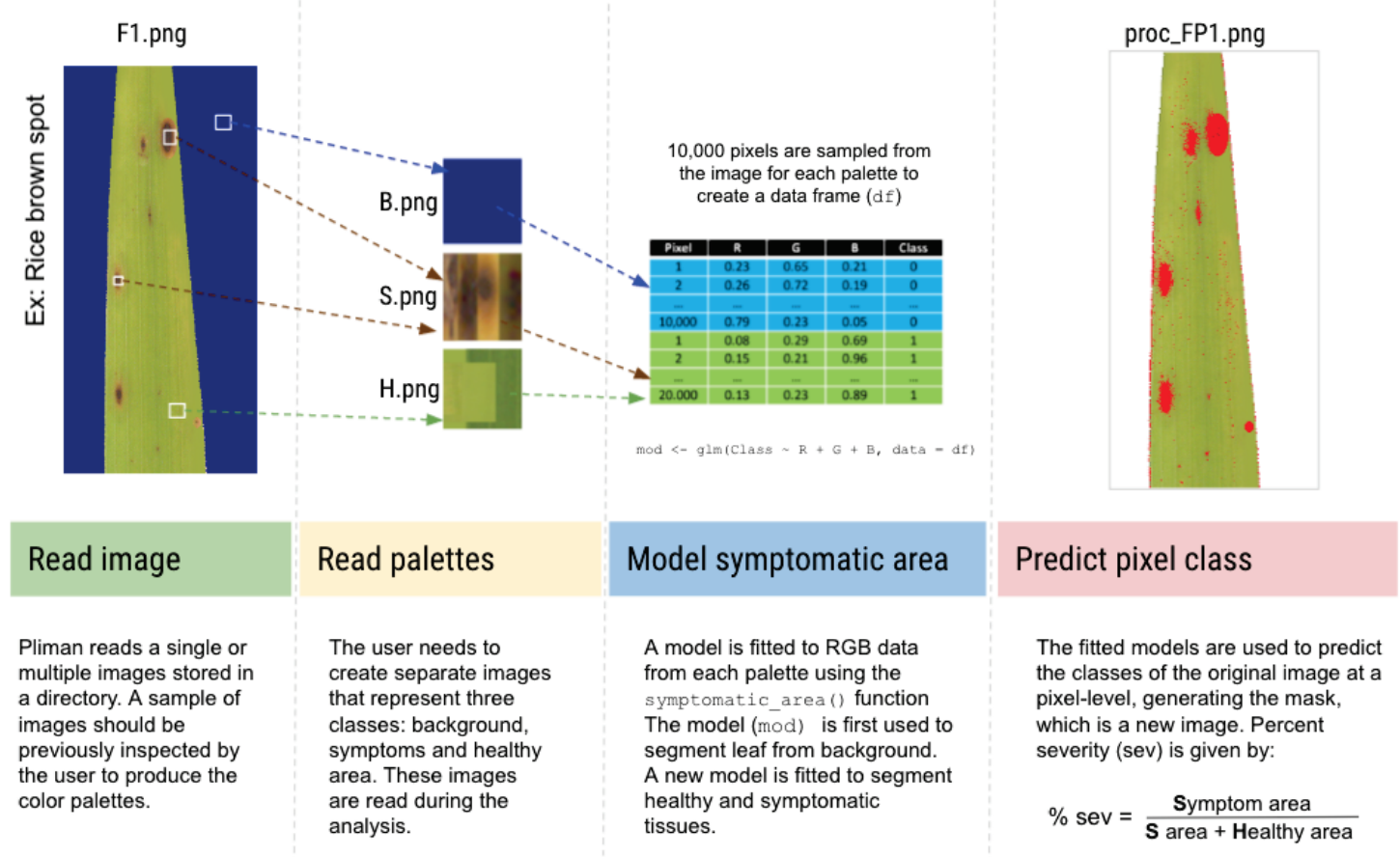

Figure 1. Details for four main steps in the workflow in pliman to compute disease severity using the symptomatic_area() function

A generalized linear model (binomial family) fitted to RGB data is first used to segment the leaf from the background. This allows computing the severity either using leaf images with a contrasting background or leaf-only images (e.g. sections of the leaf). The reference color palettes need to be produced manually by sampling small areas representative of the three sub-areas of the image to produce a composite image. An example code for importing the image to process as well as the image palettes from the current working directory, and computing severity for a single image file (as in the example figure 1 ) is shown below:

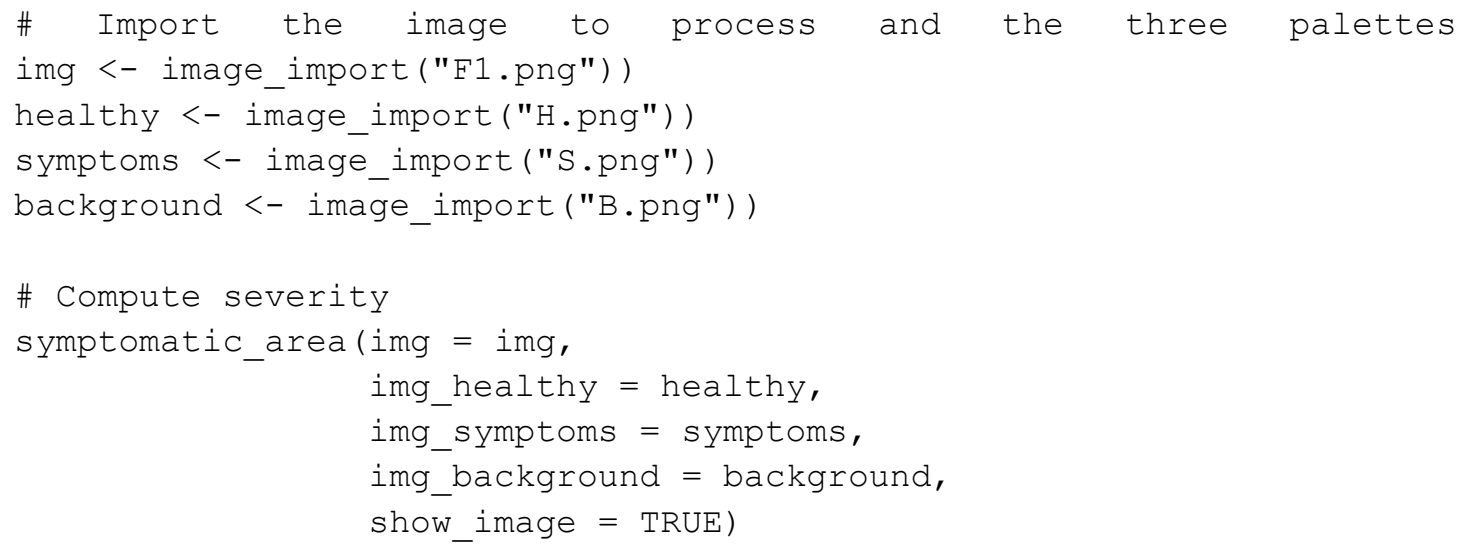




\section{\%OSFPREPRINTS \\ https://doi.org/10.31219/osf.io/jfhm7}

If the user needs to compute severity for several images, the img_pattern argument in symptomatic_area () allows for batch processing images. These images should contain a common pattern name (e.g. same initials) and that are stored in the current working directory, or subdirectory, which should be informed in the dir_original argument. To speed up the processing time, especially for a large number of images, the argument parallel = TRUE can be used. In this case, the images are processed asynchronously (in parallel) in separate R sessions running in the background for the same machine. The number of sections is set to $80 \%$ of available cores by default. This number can be controlled explicitly with the argument workers. An example code for batch processing (as in the example Figure 1 ) is shown below:

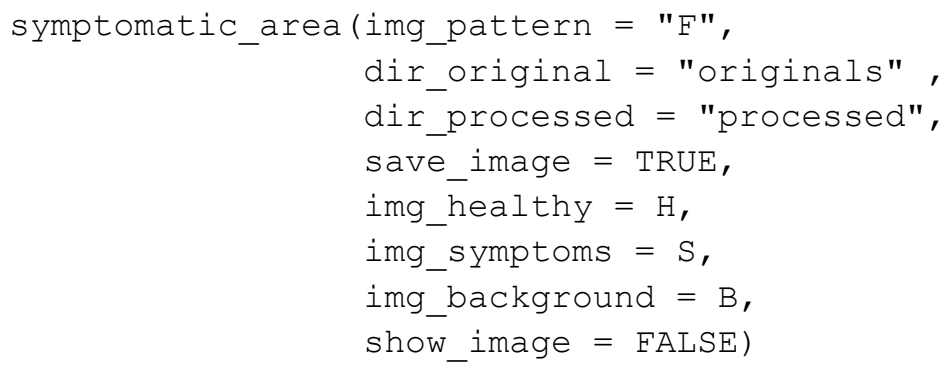

In the above example, all images that match the pattern "F" (e.g., "F1.png", Fig1.png", "F2.jpg") in the subdirectory "originals" will be processed. The argument save_image = TRUE will ensure the masks will be saved with a prefix "proc_" in the subdirectory "processed". If this argument is NULL (default) the masks will be saved into the current working directory.

\section{Validation of the pliman for severity prediction}

A first evaluation of pliman consisted in the comparison of its predictions of severity with measures obtained using a reference software, APS Assess 2.0 ${ }^{\circledR}$ (Lamari 2008) which was assumed to provide the "actual" severity. Given that the production of the color palettes is made by an individual, we hypothesized that the user producing the palettes (hereafter user) is a source of variation in the results. To test this hypothesis, four sets of sample palettes were produced as shown in Figure 2. First, each user (authors of this work) independently produced their own set of palettes. The three sets were later joined to produce a 'joint' palette. The 


\section{$\because$ OSFPREPRINTS}

https://doi.org/10.31219/osf.io/jfhm7 four sets (individuals and the joint palette) were used to compute the symptomatic area for all images, resulting in 996 severity predictions.

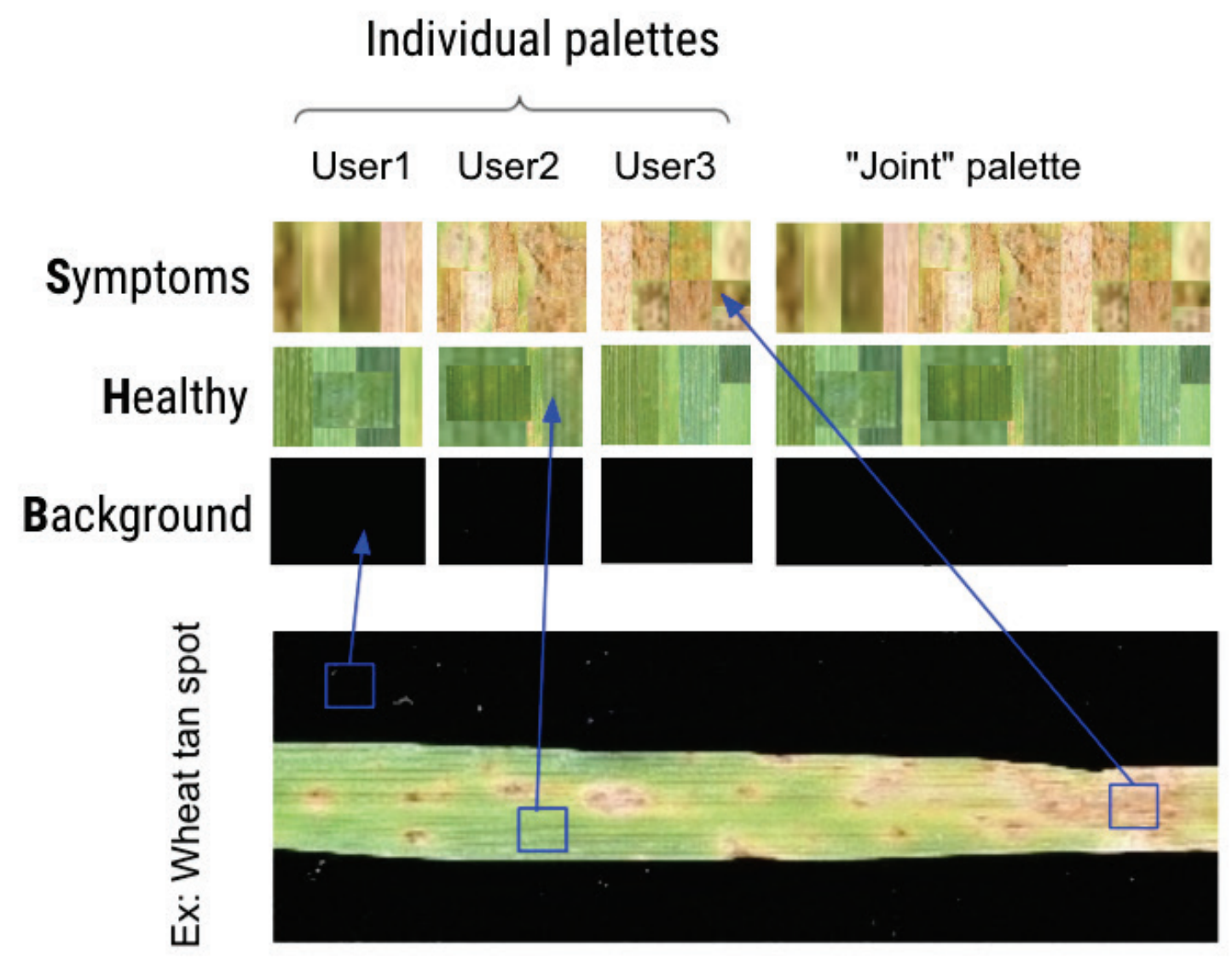

Figure 2. Palettes produced by three individual users and the "joint" palette produced by joining the three images into a single image prior to pliman predictions.

\section{Image collection}

We searched the literature on the development of standard area diagrams (SADs) and identified studies that shared publicly the datasets of symptomatic images used in the work. Five datasets were gathered from online databases and one image dataset (bean angular leaf spot) was generated for this study. We randomly selected 19 to 50 images (representing the range of severity and various symptomatic patterns) from each study to evaluate pliman predictions of severity, totaling 249 images (Table 1). Each image was carefully inspected and, in some cases, the background was standardized prior to measuring the actual severity using available software (details in the next section). 
Table 1. Information about the collections of images of symptoms of plant diseases used in this study

\begin{tabular}{|c|c|c|c|c|}
\hline Disease/crop name & N. of images & $\begin{array}{l}\text { Image } \\
\text { resolution }\end{array}$ & Device & Reference \\
\hline Bean angular leaf spot & 19 & $867 \times 1122$ & Scanner & This study \\
\hline Rice brown spot & 50 & $257 \times 3197$ & Scanner & $\begin{array}{l}\text { (Schwanck and Del } \\
\text { Ponte 2014) }\end{array}$ \\
\hline Wheat tan spot & 30 & $110 \times 768$ & Camera & (Gonçalves et al. 2020) \\
\hline $\begin{array}{l}\text { Tobacco - Xylella } \\
\text { fastidiosa }\end{array}$ & 50 & $478 \times 1022$ & Scanner & (Pereira et al. 2020) \\
\hline Olive Peacock Eye & 50 & $1082 \times 346$ & Scanner & (Sachet et al. 2017) \\
\hline Soybean Rust & 50 or $205^{b}$ & $735 \times 1152$ & Scanner & (Franceschi et al. 2020) \\
\hline
\end{tabular}

${ }^{a}$ The average resolution (widthxheight) in pixels of the analyzed images. ${ }^{b}$ The set with 205 images was used to compute the benchmark and concordance tests with different image resolutions.

\section{Measure of the reference (actual) severity}

The reference value for actual severity on each image was measured using the proprietary

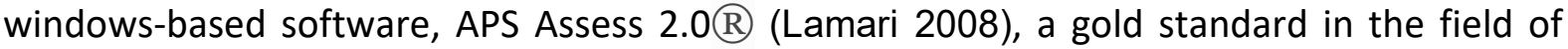
phytopathometry (Del Ponte et al. 2017). For all image datasets, the HSV model was used to separate the leaf from the background, and subsequently separate the area affected by the disease from the healthy area. Threshold levels in the manual panel were set according to each leaf and disease (Supplementary Table 1). The same user (user 3 in our study) selected the area of interest when thresholding the image to obtain the percent severity for each disease. For comparison purposes, we further determined severity (also by user 3 ) on all leaf images using two additional softwares: ImageJ (Schneider et al. 2012) and Leaf Doctor (Pethybridge and Nelson 2015). Details on the threshold limits for segmentation are also presented for these other software (Supplementary Table 1). 


\section{Agreement statistics}

Lin's concordance correlation coefficient (CCC) analysis (Lin 1989) was computed for testing the agreement between pliman-predicted and APS Assess severity for each disease and user. Further head-to-head comparison (agreement) was made between values determined by pliman (joint palette) and all three other softwares. The overall agreement $\left(\rho_{c}\right)$ combines measures of precision and accuracy to assess the fit of pairs of observations to the $45^{\circ}$ reference line (intercept $=0$, slope $=1$ ). Briefly, the $\rho_{c}$ is calculated from the Pearson correlation coefficient $(r)$, an indicator of precision, and the bias correction factor $\left(C_{b}\right)$, which measures accuracy and is the distance to the $45^{\circ}$ reference line. The $\rho_{c}$ has been recommended and widely used in studies comparing estimates with actual severity values in phytopathometry (Del Ponte et al. 2017; Braga et al. 2020; Pereira et al. 2020). The calculations were performed in the R statistical environment 4.1.0 (R Core Team 2021) using the $\mathrm{CCC}($ ) function of the DescTools package (Andri Signorell et al. 2021).

\section{Influence of image resolution and computation time}

Most devices nowadays offer resolutions that should be sufficient for detecting even small disease lesions. Even low-end mobile phones have cameras that, under well-illuminated conditions, can provide images of reasonable quality. High-resolution images obtained either by digital scanners at high dots per inch (DPI) rates (e.g., DPI > 300) or high-end digital singlelens reflex (DSLR) cameras will always provide better optical performance, allowing very small symptoms to be flawlessly outlined (Barbedo 2016). Image resolution can, however, dramatically impact the processing time since all operations are performed at a pixel level. Here, we tested the hypothesis that the reduction of image resolution reduces the processing time with no substantial impact on prediction accuracy. For such, 205 soybean rust images (Table 1) were used. Five scenarios were planned by resizing the images before processing to the following dimensions (width $\times$ height) and equivalent approximated megapixels (MP): $1367 \times 2160$ (3 MP), 1020×1620 (1.7 MP), 680×1078 (0.7 MP), 510×808 (0.4 MP), and 170×270 (0.05 MP). Image resizing was performed with the argument resize of the function symptomatic_area(). 


\section{$\because$ OSFPREPRINTS \\ https://doi.org/10.31219/osf.io/jhm7}

A benchmark study was also performed to assess the impact of image resolution on the processing time. Two processing strategies (sequential and parallel) were used. In sequential programming, the images were processed sequentially using the $\operatorname{lapply}_{\mathrm{y}}()$ approach. In parallel programming, the images were processed asynchronously (in parallel) in separate $\mathrm{R}$ sessions (10) running in the background on the same machine using the $\mathrm{R}$ base parallel package. The tests were made in an Intel(R) Core(TM) i7-9750H CPU @2.6GHz laptop with 16 GB RAM.

\section{Results}

\section{User influence on the predictions}

The pliman-predicted severity values were, in general, highly concordant $\left(\rho_{c}>0.97\right.$ in $54 \%$ of the 24 comparisons: 6 diseases $x 4$ methods) with the actual values determined by APS Assess for all diseases but the Xylella on tobacco. The users, who independently produced their image palettes, showed to be a significant source of variation, with the magnitude of variation depending on the disease. While $\rho_{c}$ did not vary significantly among the users for soybean rust and bean angular spot ( $=0.97$ to 0.99 ), predictions by the palettes produced by user 2 significantly leased accurate $\left(\rho_{c}=0.88\right.$ to 0.92$)$ than the other palettes $\left(\rho_{c}=0.99\right.$ to 1$)$ for rice brown spot and olive leaf spot. In another case, predictions by user 3 were least accurate $\left(\rho_{c}\right.$ $=0.93)$ for wheat tan spot.

Nevertheless, $\rho_{c}$ reached 0.99 using the palettes from one or another user for each disease except for the Xylella fastidiosa on tobacco. The use of the joint palette resulted in the highest levels of concordance overall (across diseases) compared to the individual users. Interestingly, the concordance between pliman predictions and APS Assess was the least when the joint palette was used for the Xylella disease. Moreover, user 3 produced a palette that resulted in significant improvement compared to the other users as well as the joint palette for this disease. 


\section{$\because$ OSFPREPRINTS}

https://doi.org/10.31219/osf.io/jfhm7

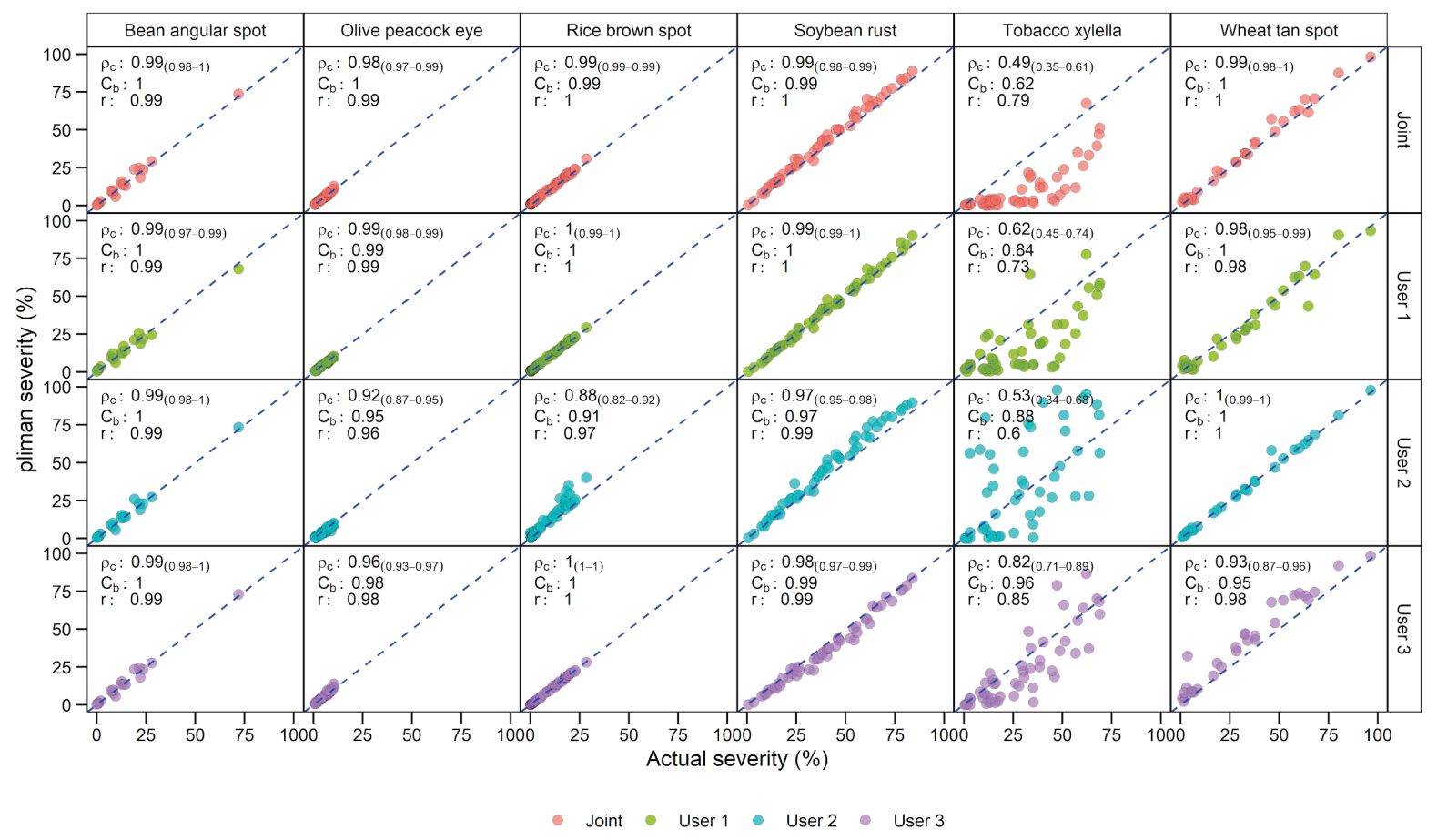

Figure 3. Relationship of measures of percent severity of bean angular spot, olive peacock eye, rice brown spot, tobacco-xylella, and wheat tan spot using APS Assess (actual severity) and pliman with four different palettes (three individuals and one joint palette). The dashed line represents the perfect concordance (1:1) line. $\rho_{c}$ (overall agreement), $C_{b}$ (bias correction factor), and $r$ (Pearson's correlation coefficient).

\section{Comparison among software}

Table 2 shows the main advantages and disadvantages of the software used in this study. LeafDoctor stands out as a mobile application with a built-in camera, which facilitates the quantification of plant diseases at the field level. The main disadvantage of APS Assess is that it requires a relatively expensive commercial license for a single user. Advantages of pliman rely on the following aspects: The first is that as an R package, it is free and open-source, allowing the user contributions to code improvements and bug corrections quickly. The second is the implementation of a batch processing strategy with parallel computation, allowing a faster process of disease quantification in several images.

In pliman, using a sequential processing strategy was 30 times faster than the quantification with APS Assess, 18 times faster than ImageJ, and 12 times faster than Leaf Doctor. When implementing a parallel strategy using images of reduced resolution, four 


\section{$\because$ OSFPREPRINTS \\ https://doi.org/10.31219/osf.io/jhm7}

images were processed per second, meaning $\sim 40$ times faster than APS Assess, 260 times faster than ImageJ, and 170 times faster than Leaf Doctor (Table 2).

Table 2. Comparison of features available in APS Assess, ImageJ, Leaf Doctor, and pliman for measuring plant disease severity on images of plants.

\begin{tabular}{|c|c|c|c|c|}
\hline Attribute & APS Assess & ImageJ & LeafDoctor & Pliman \\
\hline Free & $x$ & $\checkmark$ & $\vee$ & $\checkmark$ \\
\hline Open-Source & $x$ & $\checkmark$ & $x$ & $\checkmark$ \\
\hline Portability & $x$ & $x$ & $\checkmark$ & $x$ \\
\hline Built-in camera & $x$ & $x$ & $\checkmark$ & $x$ \\
\hline Batch processing & $\checkmark$ & $\checkmark$ & $x$ & $\checkmark$ \\
\hline Parallelization & $x$ & $x$ & $x$ & $\checkmark$ \\
\hline Processing time (s) & $100^{a}-230^{b}$ & $60^{\mathrm{a}}$ & $40^{\mathrm{a}}-46^{\mathrm{b}}$ & $0.23-3.38^{c}$ \\
\hline Accuracy & The "true' value & $0.99-1^{d}$ & $0.99-1^{d}$ & $0.62-1^{d}$ \\
\hline Precision & The "true" value & $0.99-1^{d}$ & $0.98-1^{d}$ & $0.61-0.99^{d}$ \\
\hline
\end{tabular}

\footnotetext{
${ }^{a}$ Approximate time needed to process each image in this study. ${ }^{b}$ Average results for six diseases reported by Pethybridge and Nelson (2015). ${ }^{c}$ Minimum and maximum mean values obtained in the batch processing of 205 images (See more details in Figure 6). ${ }^{d}$ minimum and maximum values obtained in this study.
}

The measures of severity obtained using the four software strongly agreed between them. The concordance coefficient values $\left(\rho_{c}\right)$ were $>0.97$ for all pairwise comparisons except for Xylella fastidiosa on tobacco (Figure 4). As expected, lower levels of concordance were obtained when pliman was compared with the three other software for that disease $\left(\rho_{c}=\right.$ $0.50)$. 


\section{$\because$ OSFPREPRINTS \\ https://doi.org/10.31219/osf.io/jhm7}

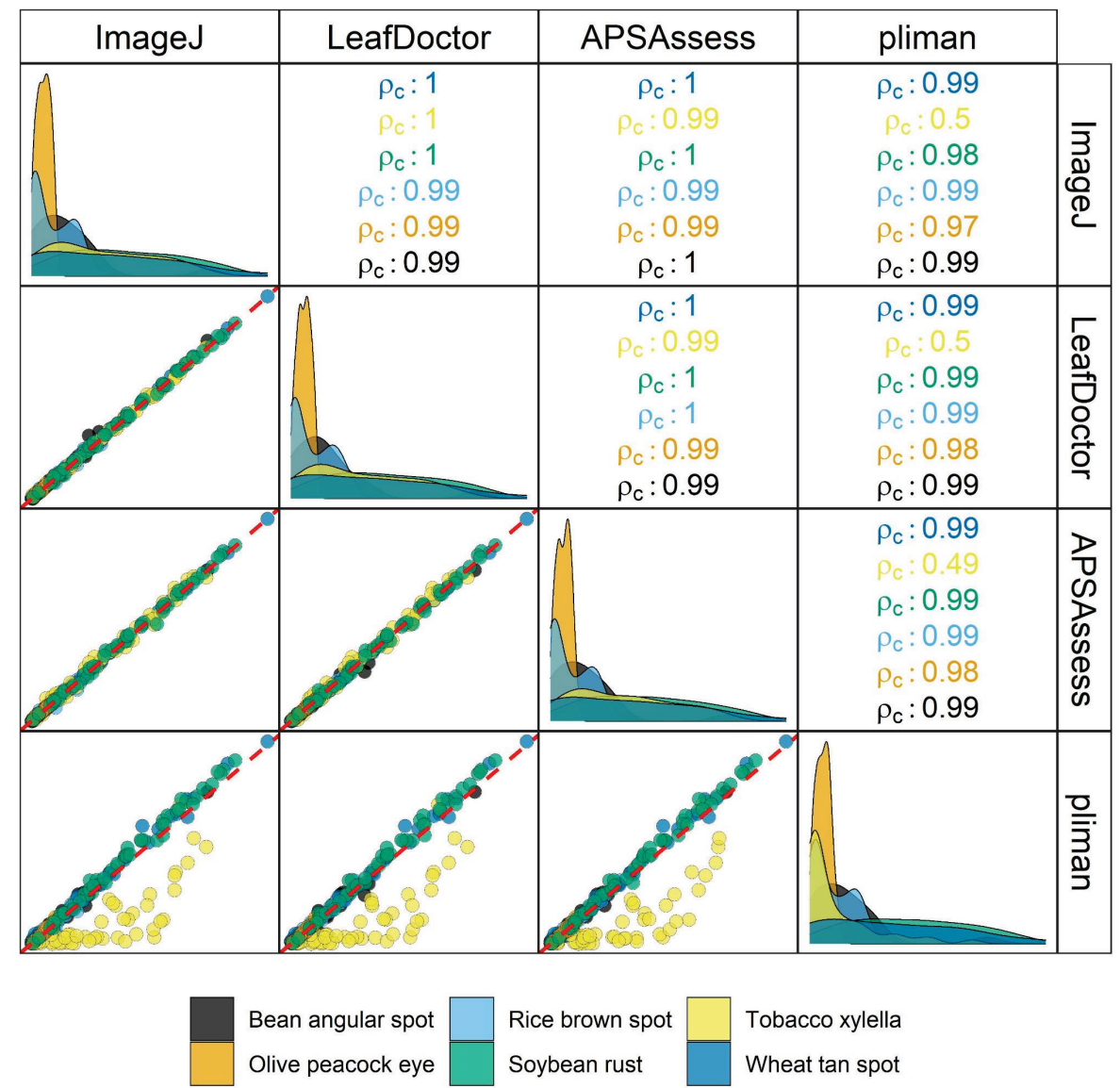

Figure 4. Relationship of measures of percent severity of Bean angular spot, Olive peacock eye, Rice brown spot, Tobacco xylella and Wheat tan spot using four different image-processing programs. The red dashed line represents the perfect concordance.

\section{Image resolution and processing time}

The image resolution showed to not be a source of variation for accuracy and precision (Figure 5). This is supported by the high values of $C_{b}(0.991$ to 0.999$)$ and $r(0.994$ to 0.997$)$ observed, respectively. A slight reduction in accuracy $\left(C_{b}\right)$ and precision $(r)$ was observed when the resolution was reduced to $0.05 \mathrm{MP}$. The higher reduction of $C_{\mathrm{b}}$ and $\rho_{\mathrm{c}}$ compared to $r$ in this image (Figure 5b) suggests that low-resolution images may show consistent values of severity that are farthest away from the "actual" values.

Prediction residuals ranged from $-12.7 \%$ to $9.43 \%$ (Figure $5 c$ ). The lower prediction accuracy observed for low-resolution images seems to be related to an underestimation of disease severity, which is evidenced by the higher density of negative prediction residuals for 


\section{\%OSFPREPRINTS \\ https://doi.org/10.31219/osf.io/jfhm7}

these images. Images with larger resolutions presented a right-skewed residual distribution (positive-skew), suggesting an overestimation of disease severity in these cases.

a

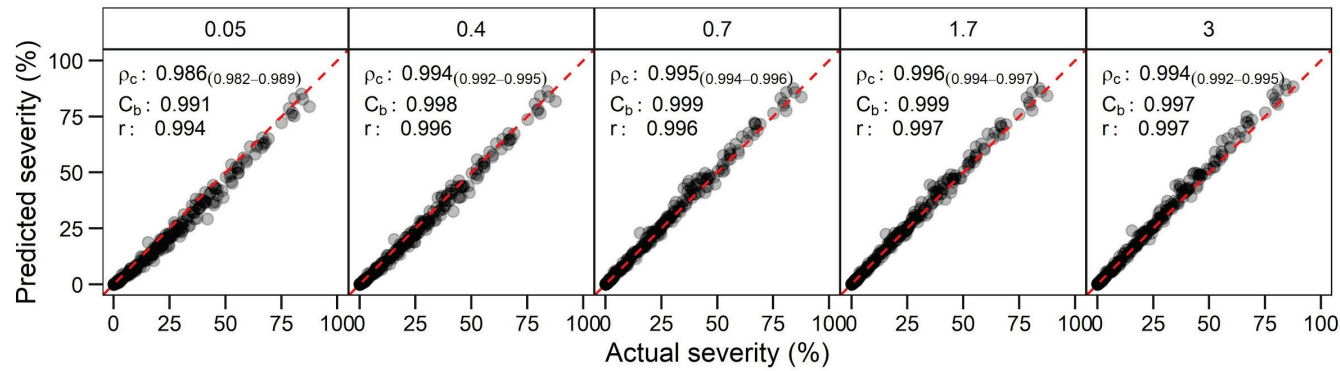

b

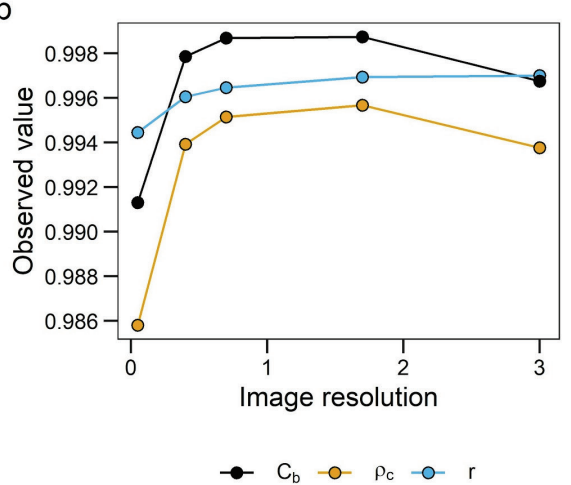

C

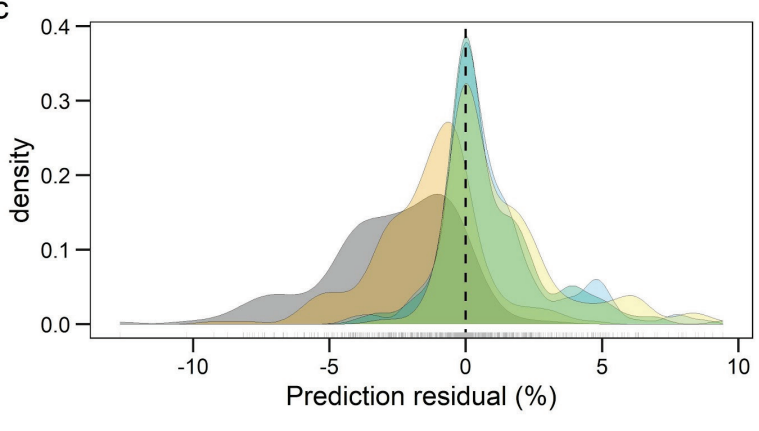

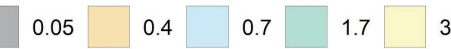

Figure 5. The effect of image resolution on prediction accuracy $\left(C_{b}\right)$, precision $(r)$, Lin's concordance coefficient $\left(\rho_{c}\right)(a-b)$, and distribution of prediction residuals $(c)$.

As expected, image resolution significantly affected processing time (Figure 6). The average time needed to process one single image using sequential strategy was reduced by $\sim 88 \%$ (from $3.38 \mathrm{~s}$ to $0.44 \mathrm{~s}$ ) when image resolution was reduced from 3 to $0.05 \mathrm{MP}$. Using parallel processing -in this case by running 10 multiple $R$ sections in the same machine- the average processing time for the same images was lesser, ranging from $1.98 \mathrm{~s}$ to $0.23 \mathrm{~s}$ (Figure 6). In our case, considering a linear trend, the reduction in processing time through reduction in the image resolution was more evident for the sequential strategy $\left(-0.95 \mathrm{~s} \mathrm{MP}^{-1}\right)$ than the parallel strategy $\left(-0.56 \mathrm{~s} \mathrm{MP}^{-1}\right)$. 


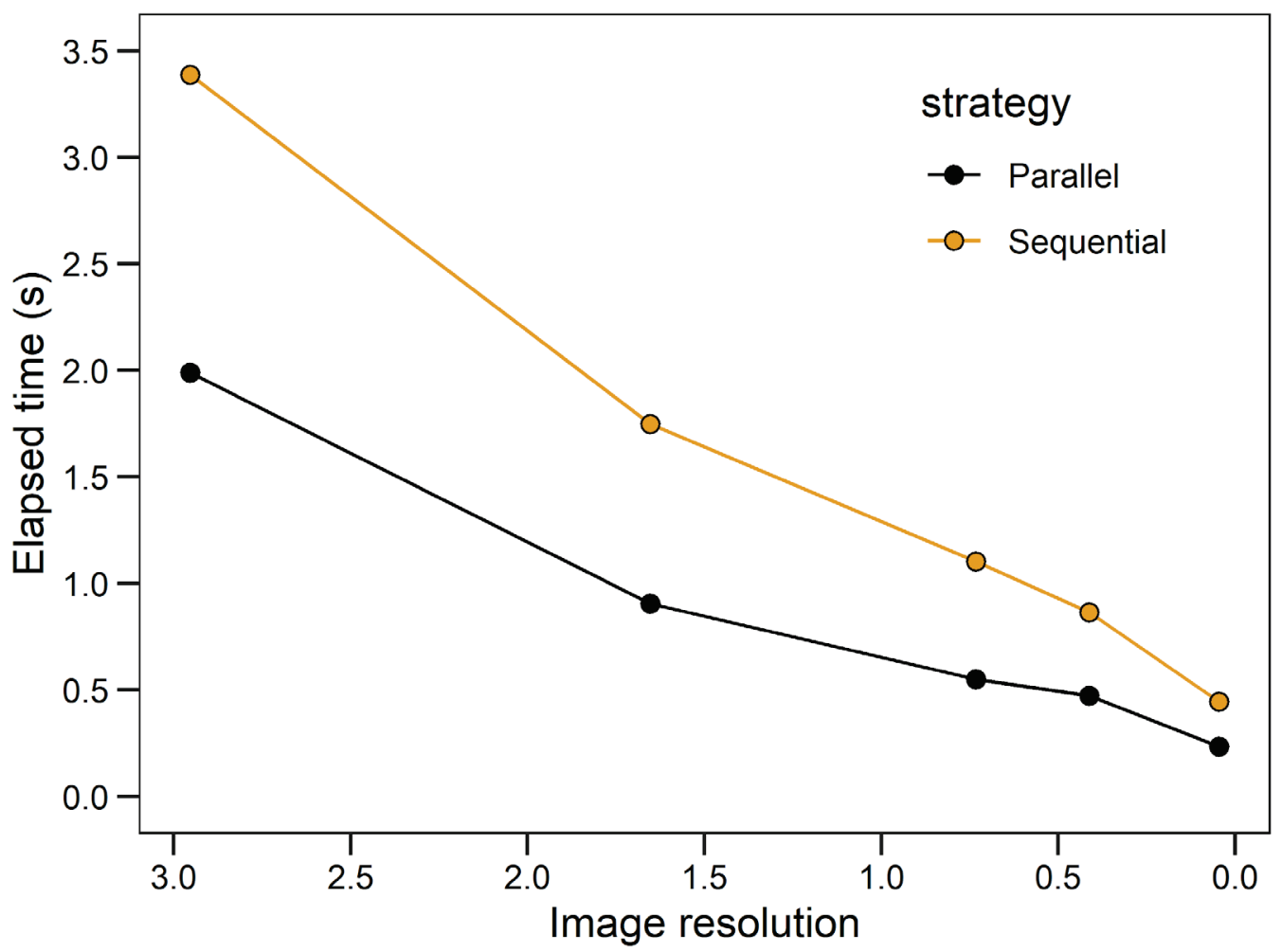

Figure 6. The effect of image resolution on processing time per image using sequential and parallel strategies. The elapsed time represents the average of 205 processed images.

\section{Discussion}

To the best of our knowledge, pliman is the first R package with built-in functions dedicated for computing disease severity expressed as percent area affected. Different from the other software compared in this study, pliman implements a model-based pixel-level prediction of classes (prior to calculating percent severity) based on the RGB information extracted from reference images (the palettes) produced by the user. Using this approach, we showed that the predictions were highly concordant with measures obtained by commonly used software aimed at performing similar tasks. In particular, the predictions of severity were highly consistent with APS Assess, which is considered a "gold standard" for thresholding disease symptoms based on color space information.

In addition to producing accurate results, the batch processing strategy implemented in pliman may be a considerable advantage. Previous studies pointed out that the processing time can vary significantly depending on the method used. Whereas manual delineation of 


\section{$\because$ OSFPREPRINTS \\ https://doi.org/10.31219/osf.io/jfhm7}

the symptomatic areas using a ruler may take several minutes, the measurements using software such as APS Assess are rather fast (Barbedo 2014). Previous studies (Pethybridge and Nelson 2015) showed that the processing time of one image using APS Assess was 230s on average (ranging from $174 \mathrm{~s}$ to $297 \mathrm{~s}$ depending on the disease) and that Leaf Doctor was substantially faster than APS Assess with an average time of $46 \mathrm{~s}$ ( $32 \mathrm{~s}$ to $50 \mathrm{~s}$ ) per image. Comparing these results with the processing time observed in this study, prediction of severity with pliman using a parallel processing strategy should be $\sim 70$ times faster than APS Assess and $\sim 14$ times faster than Leaf Doctor (Table 2). As more powerful hardware becomes available, execution times are expected to become negligible, mainly for a set with few, lowresolution images. In such cases, it is expected that parallel strategy will not provide a significant reduction in (or even increase) the processing time.

It is worth mentioning that the processing time presented in this study is not taking into account the time for preparing the color palettes. Similar to any other threshold-based software for disease quantification, the time for defining the thresholding parameters may vary depending on the characteristics of the symptoms and the ability/training of the user (Barbedo 2014; Pethybridge and Nelson 2015). If there is a strong contrast between leaf and background as well as healthy and diseased tissues (e.g., rice brown spot, Supplemental figure S2), the process of creating the required palettes might not take more than 2 or 3 minutes, the average time determined in this study. On the other hand, if the symptoms are more subtle and lesions are rather small (e.g., Xylella fastidiosa, Supplemental Figure S4), the process can take significantly longer and the results showed to vary significantly among the users and be less accurate.

When assessing plant disease severity visually, raters have been considered an important source of error and variability affecting accuracy (Bock et al. 2021). In our study, differently from estimation, the user of pliman needs to manually select areas of the image and prepare a new image that represents the range of color variations that are specific to at least two portions: the symptomatic and the non-symptomatic area displayed in the image. This is a subjective component in the process that has been shown to be a significant source of variation based on our results. It is thus important that the palettes are prepared by users who are capable of more correctly delineating the target areas of an image. Alternatively, our 


\section{$\because$ OSFPREPRINTS \\ https://doi.org/10.31219/osf.io/jfhm7}

data showed that, for five diseases, a joint palette, with portions prepared independently by three uses, produced the best results.

Most symptoms do not have well-defined edges. Instead, they gradually fade into healthy tissue (Supplementary Figures S5-S6). As a result, there is no unambiguous segmentation (Barbedo 2016). Otsu's (Otsu 1979) unsupervised threshold selection method implemented in pliman might provide a satisfactory consistency of symptoms selection across images, but even this is questionable since symptoms can also vary significantly in color, shape, and size from one image to another (Bock et al. 2008). This variation was observed for the Xylella tobacco in this study (Supplemental Figure S5) and can explain (at least in part) the lower levels of concordance between pliman and APS Assess measures for that disease for most users (Figure 3).

Even though pliman produced satisfactory results compared with state-of-art software, future improvements will be necessary. The next efforts will be focused on the implementation of alternative, unsupervised methods to improve the accuracy of symptom identification in contrasting images, such as adaptive thresholding (Wang et al. 2013; Singh and Misra 2017) and deep convolutional neural network (Boulent et al. 2019; Liang et al. 2019).

The current (0.3.0) and any future version of the pliman $\mathrm{R}$ package is compatible with all common operating systems available in stationary computers. It may serve as a useful and accurate alternative tool to accelerate studies involving disease severity measurements either on single or batches of leaf images. R users will find pliman fairly easy to use and will be surprised at how the setting of four arguments will allow processing thousands of images while they enjoy a cup of coffee!

\section{Authors' contributions}

TO and EMDP conceptualized the study. SMPA obtained the data. TO designed the software, and conducted the analysis. All authors wrote the first draft of the manuscript. EDP revised the manuscript critically. All authors read and approved the final manuscript. 


\section{Data availability statement}

https://doi.org/10.31219/osf.io/jfhm7

The datasets generated during and/or analyzed during the current study as well as the codes used to reproduce the examples are openly available in the website at https://doi.org/10.5281/zenodo.5069025

\section{Conflict of interest}

The authors declare no conflict of interest.

\section{Acknowledgments}

The first author would like to thank Dr. Alcinei Mistico Azevedo, who provided valuable suggestions on implementing image segmentation in R.

\section{References}

Andri Signorell et al. (2021) DescTools: Tools for Descriptive Statistics. R package version 0.99 .41

Barbedo JGA (2016) A review on the main challenges in automatic plant disease identification based on visible range images. Biosystems Engineering 144:52-60

Barbedo JGA (2014) An automatic method to detect and measure leaf disease symptoms using digital image processing. Plant Disease 98:1709-1716

Bock CH, Chiang K-S, Del Ponte EM (2021) Plant disease severity estimated visually: a century of research, best practices, and opportunities for improving methods and practices to maximize accuracy. Tropical Plant Pathology. https://doi.org/10.1007/s40858-021-00439-z

Bock CH, Parker PE, Cook AZ, Gottwald TR (2008) Visual rating and the use of image analysis for assessing different symptoms of citrus canker on grapefruit leaves. Plant Disease 92:530-541

Bock CH, Poole GH, Parker PE, Gottwald TR (2010) Plant disease severity estimated visually, by digital photography and image analysis, and by hyperspectral imaging. Critical Reviews in Plant Sciences 29:59-107

Boulent J, Foucher S, Théau J, St-Charles P-L (2019) Convolutional neural networks for the automatic identification of plant diseases. Frontiers in Plant Science 10:941

Braga K, Fantin LH, Roy JMT, et al (2020) Development and validation of a diagrammatic scale for the assessment of the severity of bacterial leaf streak of corn. European Journal of Plant Pathology 157:367-375

Del Ponte EM, Pethybridge SJ, Bock $\mathrm{CH}$, et al (2017) Standard area diagrams for aiding severity estimation: Scientometrics, pathosystems, and methodological trends in the last 25 years. Phytopathology 107:1161-1174 


\section{$\because$ OSFPREPRINTS}

https://doi.org/10.31219/osf.io/jfhm7

Franceschi VT, Alves KS, Mazaro SM, et al (2020) A new standard area diagram set for assessment of severity of soybean rust improves accuracy of estimates and optimizes resource use. Plant Pathology 69:495-505

Gonçalves J de P, Pinto F de A de C, Queiroz DM de, et al (2020) Deep learning models for semantic segmentation and automatic estimation of severity of foliar symptoms caused by diseases or pests. OSF Preprints. https://doi.org/10.31219/osf.io/wdb79

Lamari L (2008) Assess 2.0: Image analysis software for disease quantification. The American Phytopathological Society, Saint Paul, MN

Liang W, Zhang H, Zhang G, Cao H (2019) Rice blast disease recognition using a deep convolutional neural network. Scientific Reports 9:2869

Lin LI-K (1989) A concordance correlation coefficient to evaluate reproducibility. Biometrics 45:255-268

Madden LV, Hughes G, van den Bosch F (2007) The study of plant disease epidemics. APS Press, St. Paul

Nutter FW, Gaunt RE (1996) Recent developments in methods for assessing disease losses in forage/pasture crops. In: Pasture and forage crop pathology. John Wiley \& Sons, Ltd, pp 93-118

Nutter J Forrest, Teng P, Shokes FM (1991) Disease assessment terms and concepts. Plant Disease 75:1187-1188

Otsu N (1979) Threshold selection method from gray-level histograms. IEEE Transactions on Systems, Man, and Cybernetics 9:62-66

Pau G, Fuchs F, Sklyar O, et al (2010) EBImage-an R package for image processing with applications to cellular phenotypes. Bioinformatics 26:979-981

Pereira WEL, de Andrade SMP, Del Ponte EM, et al (2020) Severity assessment in the Nicotiana tabacum-Xylella fastidiosa subsp. pauca pathosystem: design and interlaboratory validation of a standard area diagram set. Tropical Plant Pathology 45:710-722

Pethybridge SJ, Nelson SC (2015) Leaf Doctor: a new portable application for quantifying plant disease severity. Plant Disease 99:1310-1316

R Core Team (2021) R: A Language and Environment for Statistical Computing. R Foundation for Statistical Computing, Vienna, Austria

Sachet MR, Danner MA, Citadin I, et al (2017) Standard area diagram set for olive leaf spot assessment. Ciência Rural 47:e20160923

Schneider CA, Rasband WS, Eliceiri KW (2012) NIH Image to ImageJ: 25 years of image analysis. Nature Methods 9:671-675

Schwanck AA, Del Ponte EM (2014) Accuracy and reliability of severity estimates using linear or logarithmic disease diagram sets in true colour or black and white: a study case for rice brown spot. Journal of Phytopathology 162:670-682

Singh V, Misra AK (2017) Detection of plant leaf diseases using image segmentation and soft computing techniques. Information Processing in Agriculture 4:41-49

Wang J, He J, Han Y, et al (2013) An adaptive thresholding algorithm of field leaf image. Computers and Electronics in Agriculture 96:23-39 


\section{$\%$ OSFPREPRINTS \\ https://doi.org/10.31219/osf.io/jfhm7}

Supplementary Table 1. Hue threshold levels to separate the lesion for each disease in APS

Assess and Image J.

\begin{tabular}{|c|c|c|c|}
\hline Disease/pathogen & Crop & APS ASSESS ${ }^{a}$ & ImageJ $^{\mathrm{a}}$ \\
\hline Angular Leaf Spot & Bean & $\begin{array}{c}\mathrm{L}: 31 \\
\mathrm{H}: 90-110\end{array}$ & $\begin{array}{c}\mathrm{L}: 30-45 \\
\mathrm{H}: 0\end{array}$ \\
\hline Brown Spot & Rice & $\begin{array}{c}\text { L: } 31 \\
H: 101\end{array}$ & $\begin{array}{c}\mathrm{L}: 36-42 \\
\mathrm{H}: 0\end{array}$ \\
\hline Tan Spot & Wheat & $\begin{array}{c}L: 31 \\
H: 101-110\end{array}$ & $\begin{array}{c}L: 40-50 \\
H: 0\end{array}$ \\
\hline Xylella fastidiosa & Tobacco & $\begin{array}{c}\mathrm{L}: 31 \\
\mathrm{H}: 95-105\end{array}$ & $\begin{array}{c}\text { L: } 40-46 \\
\text { H: } 0\end{array}$ \\
\hline Olive Peacock Eye & Olive & $\begin{array}{c}\mathrm{L}: 31 \\
\mathrm{H}: 90-101\end{array}$ & $\begin{array}{c}L: 35-40 \\
H: 0\end{array}$ \\
\hline Soybean Rust & Soybean & $\begin{array}{c}\text { L: } 31 \\
\text { H: 95-105 }\end{array}$ & $\begin{array}{c}\text { L: } 36-46 \\
H: 0\end{array}$ \\
\hline
\end{tabular}

${ }^{a} \mathrm{~L}$ is the lower limit and $\mathrm{H}$ is the higher limit used, which varies according to disease severity of each leaf. 


\section{$\because$ OSFPREPRINTS}

\section{Supplemental figures}

https://doi.org/10.31219/osf.io/jhm7
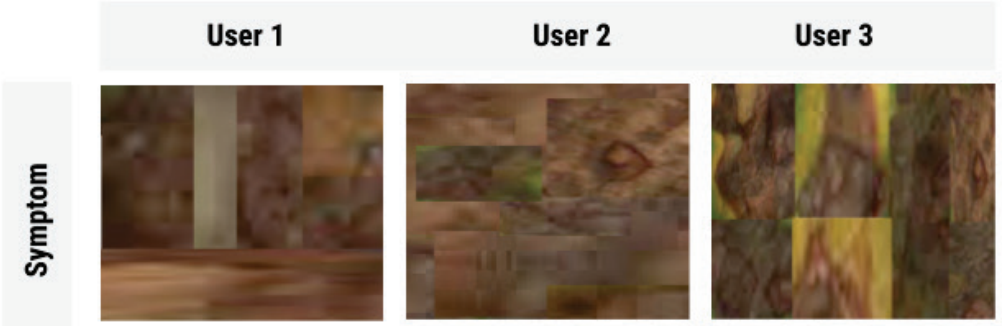

$$
\begin{aligned}
& \text { Bean angular spot } \\
& 19 \text { images }
\end{aligned}
$$
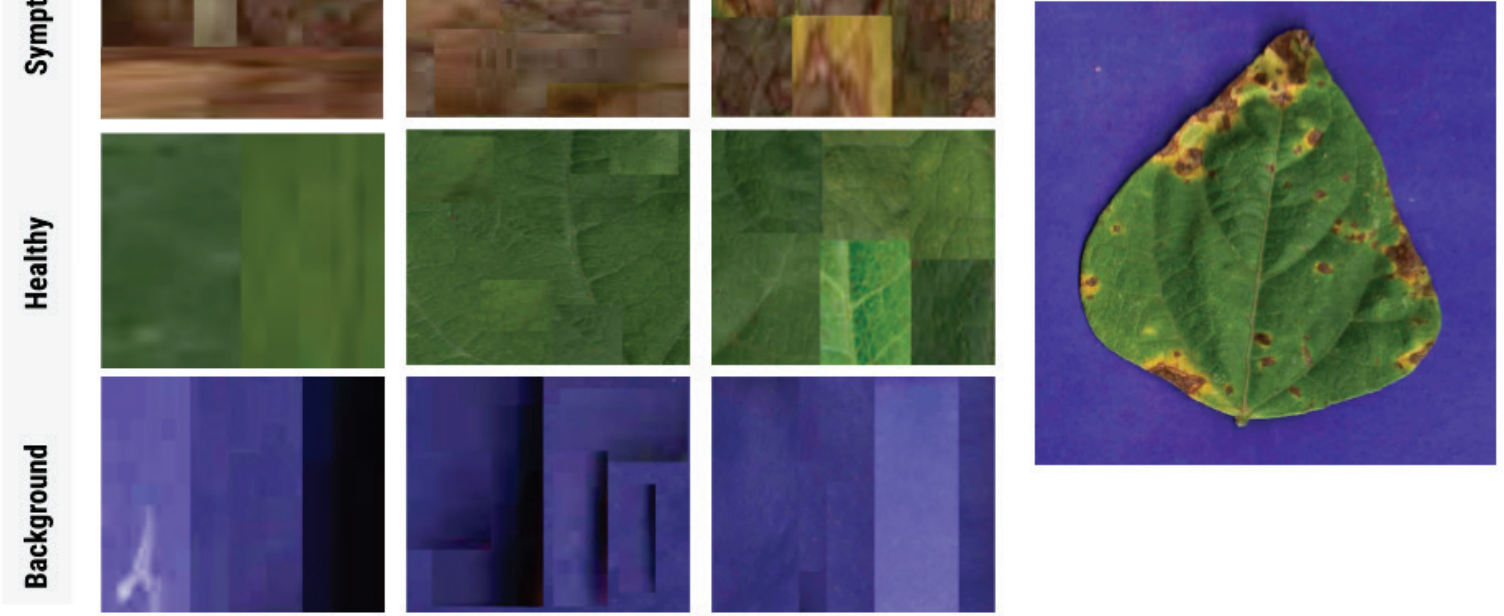

Supplemental Figure S1. The color palettes prepared by the three users (left) and an image example for the bean angular spot disease (right).

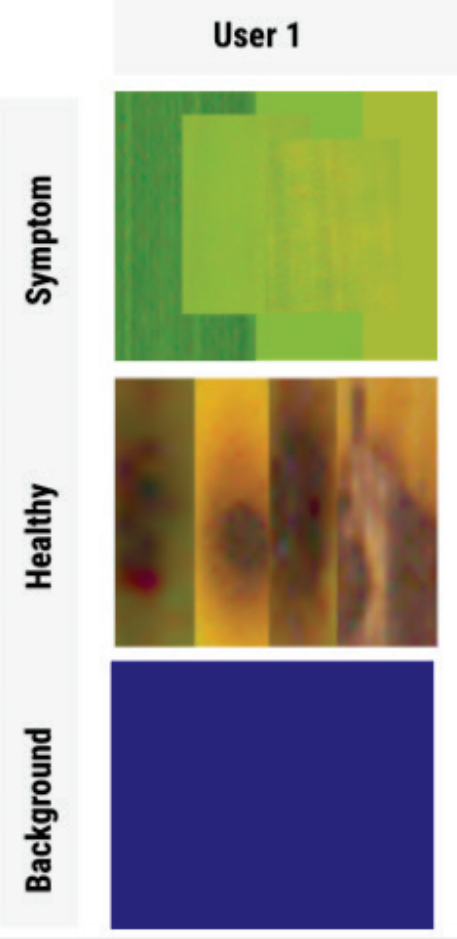

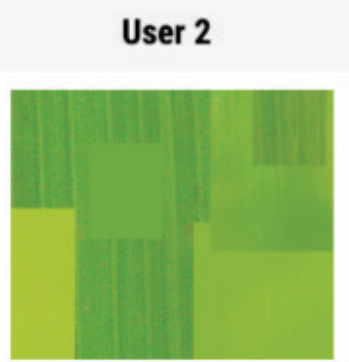
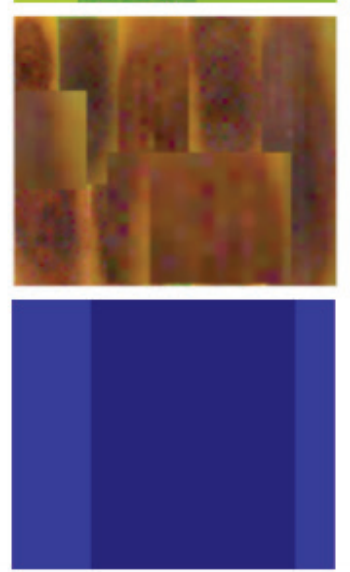

User 3

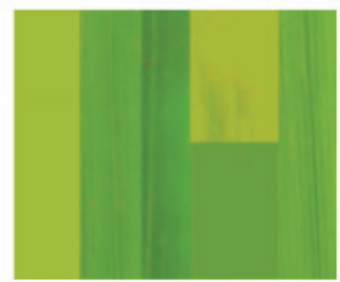

Rice brown spot

50 images
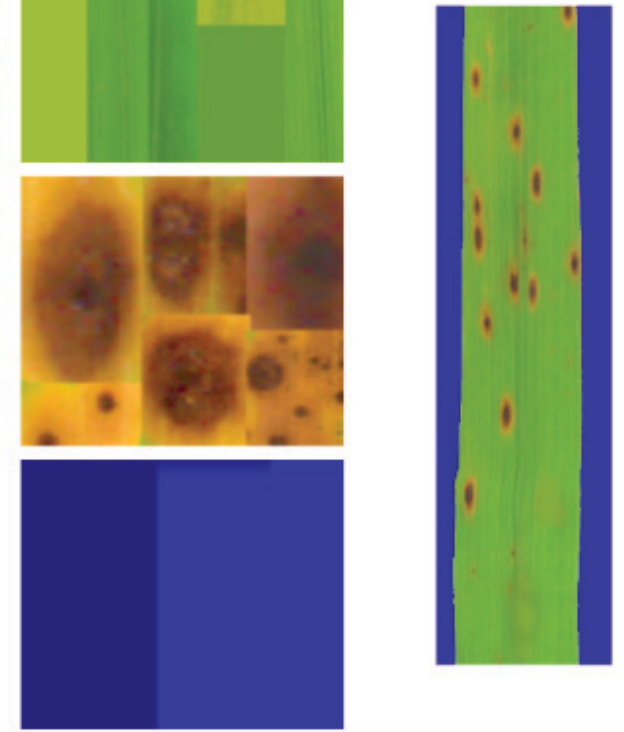

Supplemental Figure S2. The color palettes prepared by the three users (left) and an image example for the rice brown spot disease (right). 


\title{
$\because$ OSFPREPRINTS
}

https://doi.org/10.31219/osf.io/jfhm7
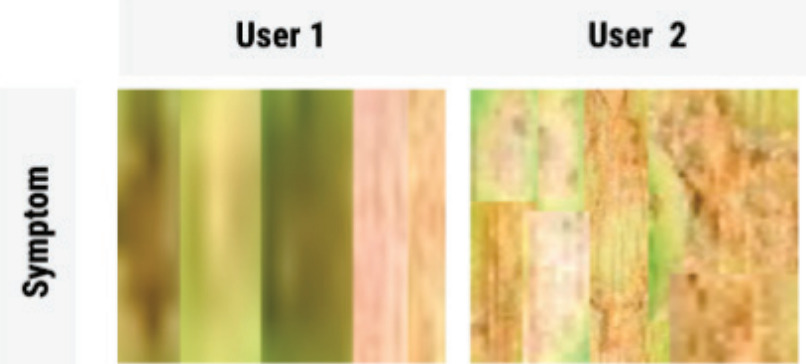

User 3
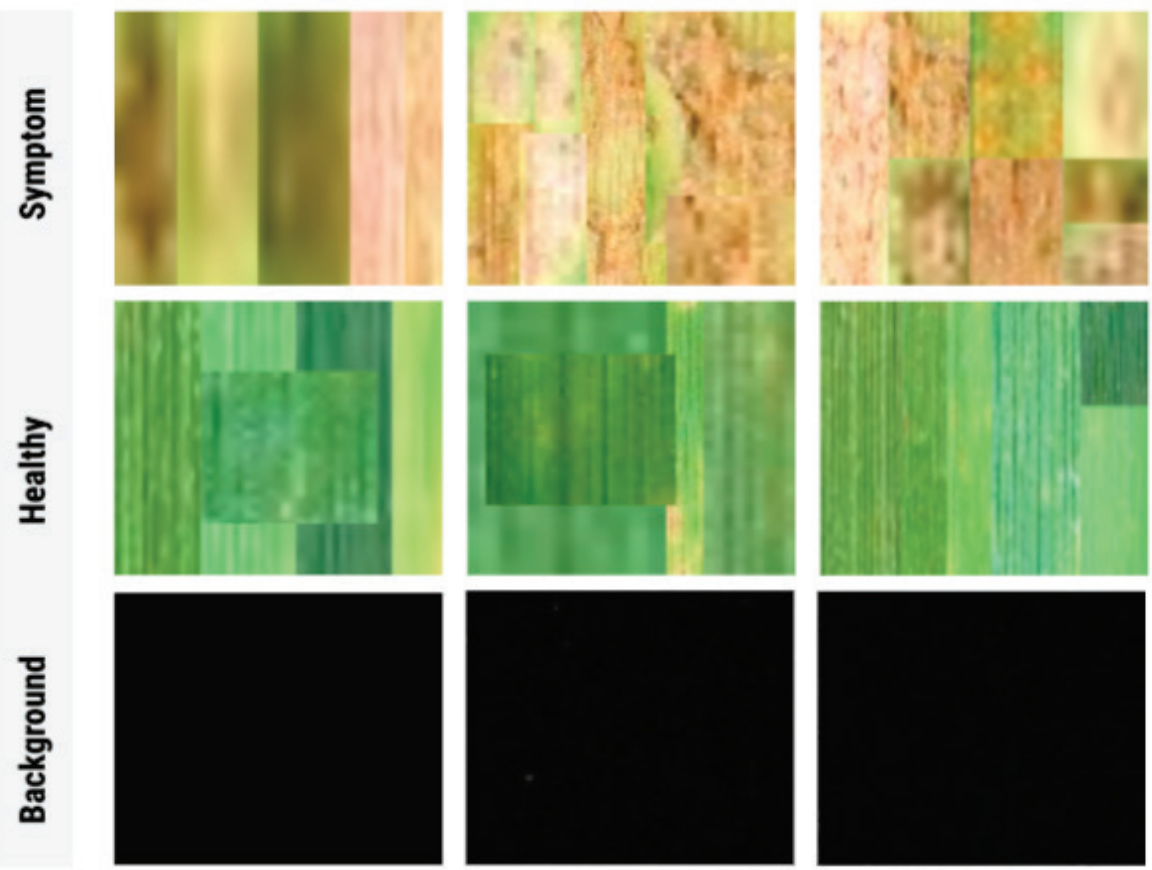

Wheat tan spot

30 images

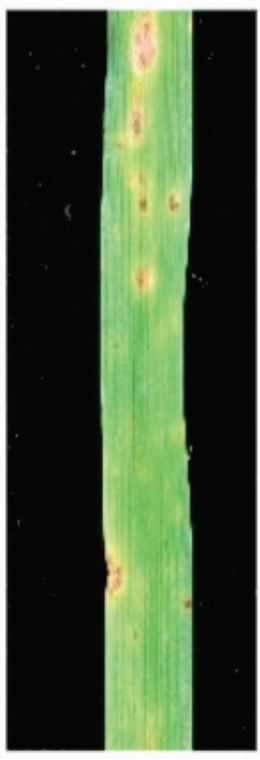

Supplemental Figure S3. The color palettes prepared by the three users (left) and an image example for wheat tan spot disease (right).

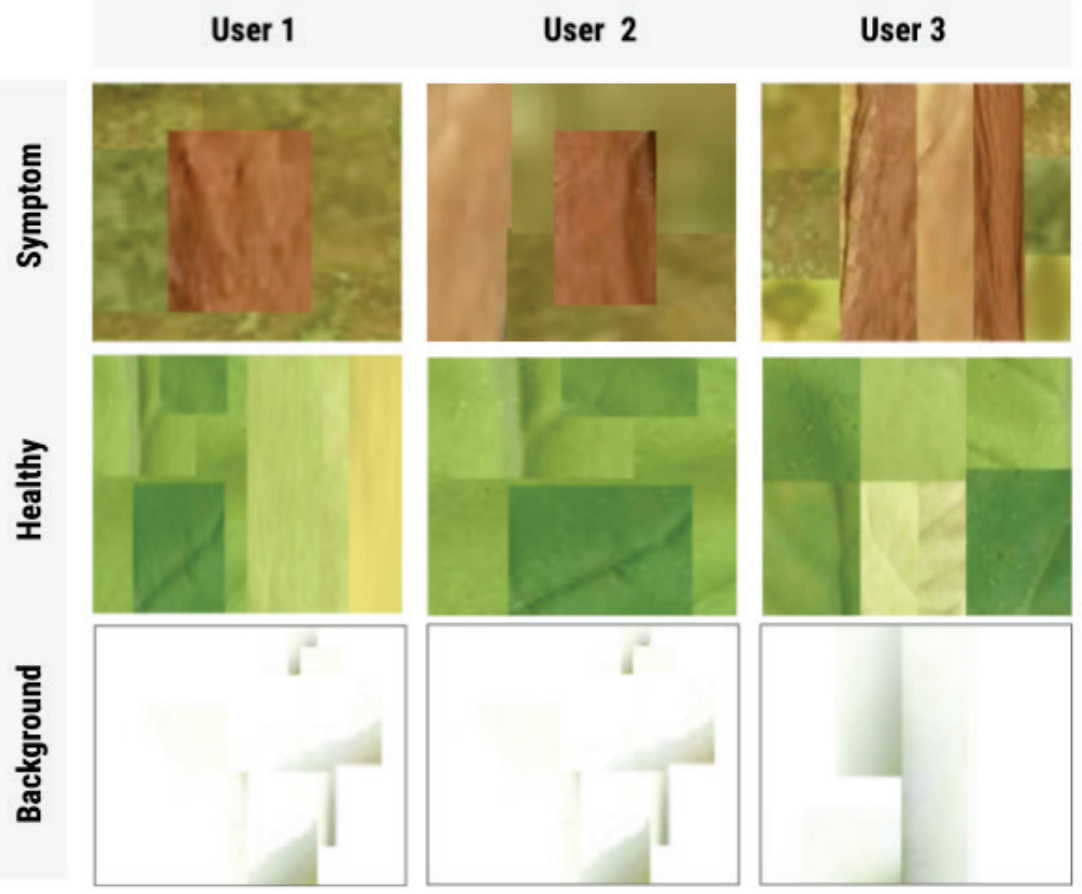

\author{
Xylella fastidiosa-tobacco \\ 50 images
}

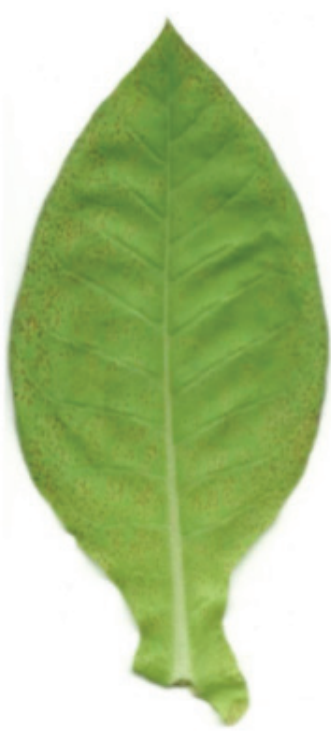

Supplemental Figure $\mathbf{S 4}$. The color palettes prepared by the three users (left) and an image example for xylella fastidiosa (right). 


\section{$\because$ OSFPREPRINTS}
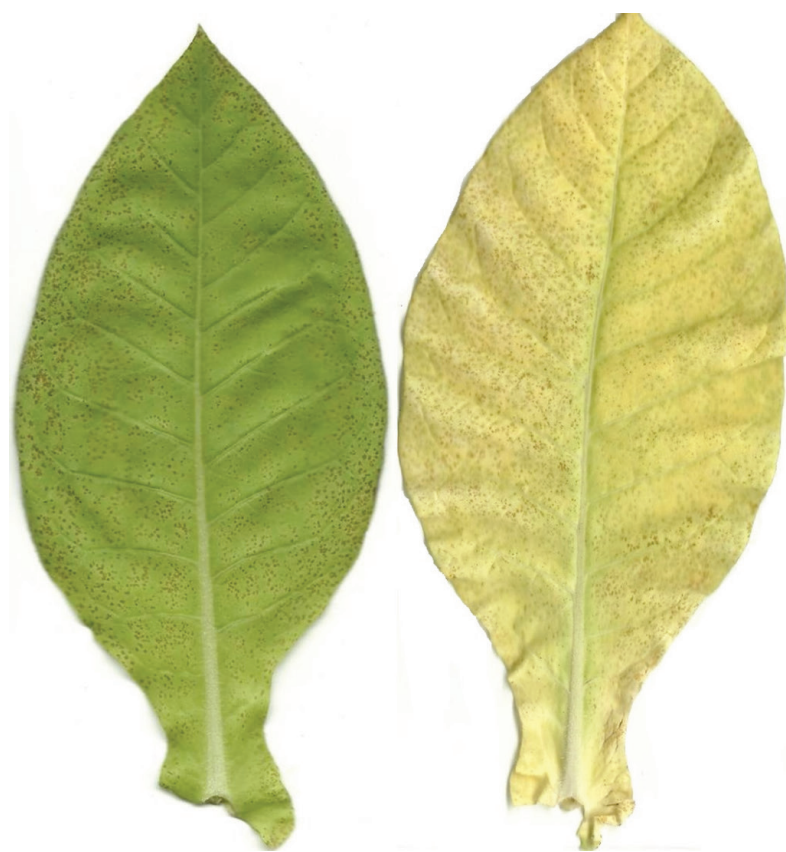

https://doi.org/10.31219/osf.io/jfhm7

Supplemental Figure S5. Four tomato leaves showing the variability of symptoms caused by Xylella fastidiosa.
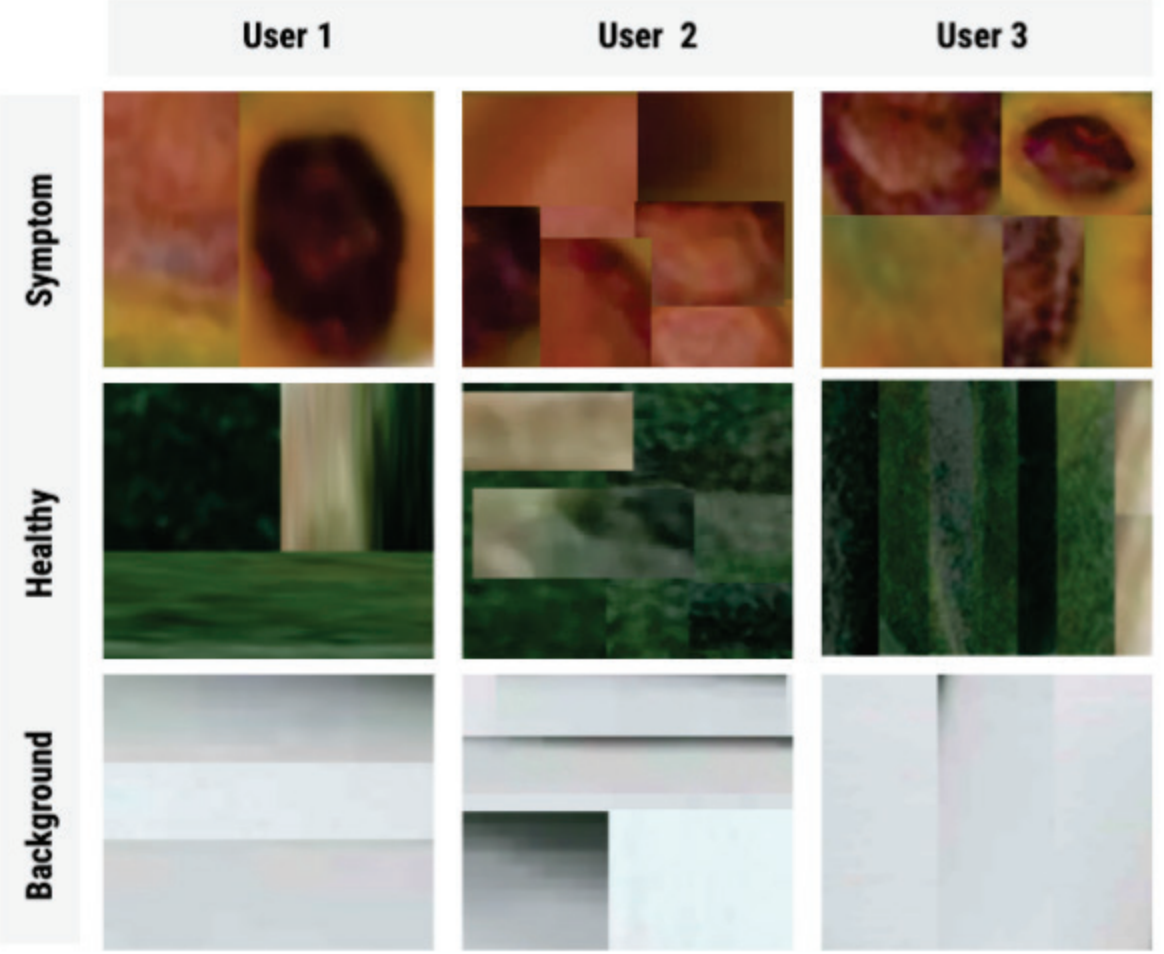

Olive peacock eye 50 imagens

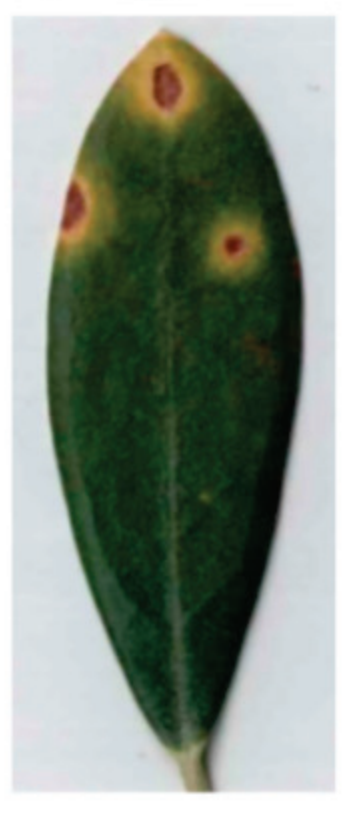

Supplemental Figure S6. The color palettes prepared by the three users (left) and an image example for olive peacock eye (right). 


\section{OSFPREPRINTS \\ https://doi.org/10.31219/osf.io/jfhm7}

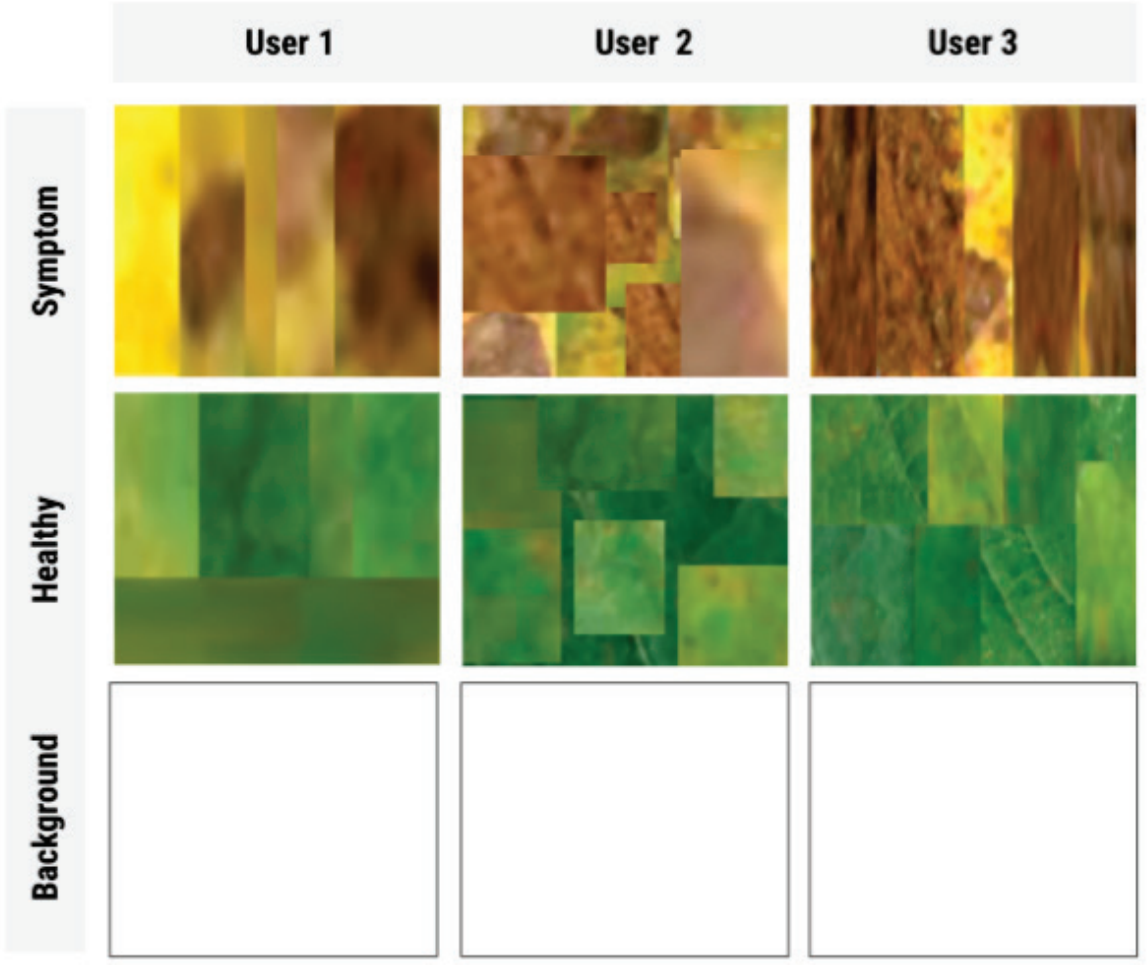

Soybean rust

50 images

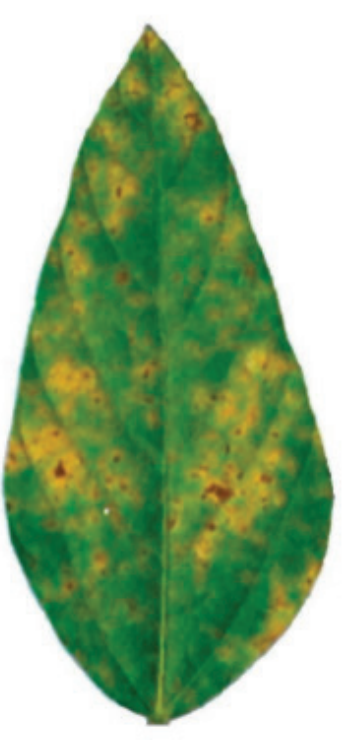

Supplemental Figure S7. The color palettes prepared by the three users (left) and an image example for the soybean rust (right). 